\title{
Efectos de la pandemia por COVID-19 en la salud mental de la población trabajadora
}

\section{Effects of the Covid-19 Pandemic on Workers' Mental Health}

\author{
Carlos Ruiz-Frutos ${ }^{1,2}$ \\ Juan Gómez-Salgado ${ }^{1,2}$ \\ 1Departamento de Sociología, Trabajo Social y Salud Pública. Facultad de Ciencias del Trabajo. Uni- \\ versidad de Huelva, España. \\ ${ }^{2}$ Universidad Espíritu Santo, Guayaquil, Ecuador.
}

Fechas · Dates

Recibido: 2020.11 .03

Aceptado: 2020.12 .08

Publicado: 2021.01.21
Correspondencia $\cdot$ Corresponding Author

\section{Juan Gómez-Salgado}

Departamento de Sociología, Trabajo Social y Salud Pública. Facultad de Ciencias del Trabajo. Universidad de Huelva. Avda. Tres de Marzo, S/N, 21071 Huelva (España). Email: salgado@uhu.es 
El impacto de la COVID-19 en la sociedad no es un tema baladí. Desde el inicio de la pandemia, el Johns Hopkins Coronavirus Resource Center había reportado hasta el 23 de noviembre de 2020 un total de 58.696 .029 personas contagiadas y 1.556.730 muertes de personas diagnosticadas con el SARS-CoV-2 (en inglés, severe acute respiratory syndrome coronavirus 2; en español, coronavirus tipo 2 del síndrome respiratorio agudo grave). Siendo España el sexto país en número de contagios (1.556.730) y el noveno en muertes (42.619) ${ }^{(1)}$.

Pese a que se trata de una crisis sanitaria, el resto de ámbitos subyacentes (social, económico, cultural, político, laboral, entre otros) están sintiendo el embate y la magnitud de una enfermedad desconocida hasta la fecha, en mayor o en menor medida, en todos los países del mundo. Como manifiesta la Organización Internacional del Trabajo (OIT) "además de ser una amenaza para la salud pública, las perturbaciones a nivel económico y social ponen en peligro los medios de vida a largo plazo y el bienestar de millones de personas"(2).

La sociedad se ha visto abocada a adoptar medidas drásticas para prevenir el contagio, como son la higiene continua de manos y superficies, la identificación y rastreo de contactos, el uso de mascarillas o el distanciamiento social, medida esta última con especial repercusión psicológica. El Consejo General de la Psicología de España (2020) ha vaticinado que unos diez millones de españoles pueden llegar a estar en riesgo de presentar problemas psicológicos derivados de la pandemia ${ }^{(3)}$. Problemas como el aislamiento domiciliario, el temor por contraer la enfermedad y la incertidumbre podían hacer que las personas desarrollaran sentimientos de miedo, ansiedad, tristeza, pensamientos suicidas, baja motivación, mayor consumo de tabaco y alcohol, etc. Además, por experiencias previas de otras epidemias, esta sintomatología no siempre desaparece cuando acaba el problema ${ }^{(4)}$.

Esta problemática se puede ver exacerbada por el impacto que causa la pandemia a nivel laboral, en la que muchas empresas han tenido que echar el cierre, muchos trabajadores se han visto inmersos en Expedientes de Regulación Temporal de Empleo (ERTE), y gran número de trabajadores han perdido su puesto de trabajo o tienen unas perspectivas de empleo no muy halagüeñas en un futuro próximo. Como sabemos, tanto la inseguridad laboral, la pérdida económica y el desempleo pueden tener graves consecuencias en la salud mental(5).

Los sanitarios han visto cómo sus compañeros y compañeras se han contagiado por el SARS-CoV-2, han fallecido personas en la más triste soledad sin poder ofrecerles cuidados humanizados, otros han sido víctima de agresión o de estigmatización social, y en gran medida ha visto que sus cargas laborales han sobrepasado los recursos personales. Han manifestado carecer de los suficientes recursos materiales para poder tratar a los pacientes con plenas garantías de seguridad y, como consecuencia, la salud mental de estos profesionales se ha visto profundamente alterada. Todo ello ha elevado los niveles de estrés, ansiedad y depresión, afectando al ejercicio de sus funciones profesionales, disminuyendo las capacidades de atención, comprensión y toma de decisiones. Ellos, sin embargo, pese a todos estos condicionantes negativos, no han dejado de prestar sus servicios ${ }^{(6)}$. 
Cuando existe una sobrecarga emocional ante la exposición al sufrimiento ajeno de forma continuada en el tiempo, como es el caso, se puede presentar sintomatología y signos afines a la fatiga por compasión. Esta problemática además de los riesgos psicosociales anteriormente expuestos, puede reducir el rendimiento laboral de las personas afectadas, haciendo del mismo menos efectivo y menos productivo, llegando en algunos casos a plantear dejar el trabajo o abandonar la profesión $n^{(7)}$.

Se ha sugerido que el impacto en la salud mental de los profesionales sanitarios, durante la primera ola de COVID-19, puede haber reducido la capacidad de resiliencia ante las futuras oleadas ${ }^{(8)}$. Se han realizado estudios en dicho colectivo, durante dicha fase crítica, arrojando valores cercanos al $40 \%$ en la subdimensión agotamiento emocional; a lo que habría que sumar que 9 de cada 10 profesionales consideraban necesaria una atención psicológica para ellos en los centros de trabajo(9). Todas estas cifras se ven incrementadas en los profesionales sanitarios que están en primera línea frente al SARS-CoV-2.

Nuestro grupo de investigación viene desarrollando el proyecto IMPACTCOVID-19 que analiza los efectos en la salud mental y que actualmente se está desarrollando en 16 países, incluido España: Argentina, Brasil, Cabo Verde, Chile, Colombia, Costa Rica, Ecuador, El Salvador, Italia, Malasia, México, Nicaragua, Paraguay, Perú y Portugal. Al utilizar el mismo instrumento, adaptado a cada país, nos facilitará la comparación de los efectos de la pandemia, aunque siendo conscientes de que la evolución de la enfermedad y las medidas preventivas adoptadas no han sido las mismas en todos los países. Se ha analizado al conjunto de la población general, a los trabajadores no sanitarios y a los trabajadores sanitarios. Dentro del colectivo de sanitarios también se estudió por separado a los especialistas en medicina y enfermería del trabajo, para lo que se contó con la colaboración de la Asociación de Especialistas en Medicina del Trabajo y la de Especialistas en Enfermería del Trabajo, con un nivel de respuesta del $42,0 \%$ y $38,8 \%$ de sus afiliados, respectivamente.

En el colectivo de los sanitarios, en la primera fase de la pandemia, hemos encontrado tasas de distrés psicológico (Cuestionario de Salud General de Goldberg GHQ-12 $\geq 3$ ) en el 80,6\% de los profesionales sanitarios del ámbito asistencial, con niveles más elevados en atención especializada (82.2\%) en comparación con atención primaria $(77.3 \%)^{(10)}$, siendo inferior en el conjunto de la población $(71.98 \%)^{(11)}$, y más baja en el subgrupo de trabajadores no sanitarios (65.1\%)(12). En todos los casos el distrés psicológico es mayor en las mujeres y los más jóvenes.

Los trabajadores sanitarios han sido el colectivo más estudiado, pero no han sido los únicos que siguieron trabajando durante el confinamiento. Dentro del colectivo de trabajadores no sanitarios hay que distinguir dos grupos: los trabajadores de actividades esenciales y los no esenciales. Los primeros han seguido su actividad laboral normal, en contacto con personas que podían estar contagiadas y con el peligro añadido de contagiar a sus familiares al volver al domicilio. Entre ellos encontramos a trabajadores de seguridad, limpieza, cuidado de personas mayores o con discapacidad, producción, distribución y venta de productos, etc. 
En el segundo grupo están los trabajadores en puestos no esenciales, que en gran medida han tenido que seguir trabajando en sus domicilios mediante teletrabajo y, en caso de no poder hacerlo, tuvieron que interrumpir temporalmente su actividad profesional por no haber podido virtualizarla. Siendo conocidos los riesgos para la salud derivados del teletrabajo, con aumento de las tensiones a consecuencia del aislamiento, la adaptación a nuevas formas de trabajar y a un nuevo entorno, que suele provocar aumento de la carga mental y familiar ${ }^{(13)}$, o factores positivos como no tener que desplazarse al trabajo(14), así como los efectos de la pérdida de empleo o la inseguridad para mantenerlo.

Se ha constatado que el entorno laboral tiene un fuerte impacto en la salud mental de los trabajadores, especialmente en situaciones límites de alta exigencia profesional. El sentido de la coherencia (SOC) es entendido como la capacidad de las personas para percibir una situación estresante como comprensible, manejable y significativa, lo que les permite utilizar sus recursos para afrontarla de forma eficaz $^{(15)}$. Se ha asociado niveles bajos de SOC en sanitarios con niveles altos de angustia psicológica y la presencia de síntomas de COVID-19(10). Esta asociación también es vista en los trabajadores no sanitarios, a lo que hay que sumar el efecto del engagement laboral, entendido como un estado de salud mental positivo y satisfactorio relacionado con el trabajo. Niveles bajos de SOC y de engagement laboral, han demostrado ser factores protectores ante la presencia de distrés, ayudando en el afrontamiento de esta complicada situación ${ }^{(16)}$. Entre las variables que predicen el distrés psicológico en los trabajadores no sanitarios encontramos ser mujer, tener una vivienda interior, una mala percepción de salud durante los últimos 14 días, el número de síntomas, haber estado en contacto con personas o material contaminado y ser trabajador autónomo ${ }^{(12)}$.

Otro aspecto interesante a analizar son las vías de información recibida sobre la pandemia y sus efectos en desarrollar distrés. Hemos constatado que, en el conjunto de la población estudiada, las redes sociales ocupan el primer lugar (77.5\%), seguidas de la televisión (58.4\%) y de las páginas webs de sociedades científicas $(48.8 \%)^{(11)}$. En los trabajadores no sanitarios se utilizaron las mismas fuentes de información, aunque eran consideradas de menor calidad y utilidad que las fuentes oficiales. El tiempo informándose y su nivel de conocimiento se asoció al nivel de distrés, existiendo mayor confianza en los profesionales sanitarios que en el sistema sanitario, y siendo la principal preocupación de los trabajaban fuera de casa el contagiar a familiares ${ }^{(17)}$. La oportunidad de ofrecer información de calidad, veraz y actualizada en Internet ha motivado a los organismos públicos, universidades e incluso editoriales de revistas de pago a crear webs específicas ofertando dicha información accesible y gratuita ${ }^{(1,18-20)}$.

Parece evidente la necesidad de preparar recursos que permitan responder a lo que la mayoría de los encuestados demanda, la conveniencia de recibir apoyo psicológico, no solo los trabajadores sanitarios y no sanitarios, sino también los enfermos y sus familiares. 


\section{Referencias}

1. Johns Hopkins University. COVID-19 Dashboard by the Center for Systems Science and Engineering (CSSE) at Johns Hopkins. [citado: 26 noviembre 2020]. Disponible en: https://coronavirus.jhu.edu/map.html.

2. Organización Internacional del Trabajo (OIT). COVID-19 y el mundo del trabajo. Ginebra: Organización Internacional del Trabajo; 2020. [citado: 30 noviembre 2020]. Disponible en: https://www.llo.org/global/topics/coronavirus/lang--es/index.htm

3. Consejo General de la Psicología de España. Más de diez millones de españoles en riesgo de presentar problemas psicológicos derivados de la pandemia de COVID-19. Madrid: Consejo General de la Psicología de España; 2020. [citado: 30 noviembre 2020]. Disponible en: https://www.copmadrid.org/web/comunicacion/comunicado/234/mas-de-diez-millones-de-espanoles-en-riesgo-de-presentar-problemas-psicologicos-derivados-de-la-pandemia-de-covid-19.

4. Wang YX, Guo HT, Du XW, Song W, Lu C, Hao WN. Factors associated with posttraumatic stress disorder of nurses exposed to corona virus disease 2019 in China. Medicine (Baltimore). 2020;99:e20965

5. International Labour Organization. Managing work-related psychosocial risks during the COVID-19 pandemic - Geneva: ILO 2020. [citado: 30 noviembre 2020]. Disponible en: https://www.lo.org/global/topics/safety-and-health-at-work/resources-library/publications/WCMS_748638/lang--en/index.htm.

6. García-Iglesias JJ, Gómez-Salgado J, Martín-Pereira J, Fagundo-Rivera J, Ayuso-Murillo D, Martínez-Riera JR, Ruiz-Frutos C. Impacto del SARS-CoV-2 (Covid-19) en la salud mental de los profesionales sanitarios: una revisión sistemática. Rev Esp Salud Pública. 2020; 94: 23 de julio e202007088

7. Boyle DA. Fatiga por compasión: el precio de la atención. Nursing. 2016;33(2):1619. doi: 10.1016/j.nursi.2016.04.010

8. Salazar de Pablo, G., Vaquerizo-Serrano, J., Catalan, A., Arango, C., Moreno, C., Ferre, F., Shin, Jl., Sullivan, S., Brondino, N., Solmi, M. y Fusar-Poli, P. "Impact of coronavirus syndromes on physical and mental health of health care workers: Systematic review and meta-analysis". Journal of Affective Disorders 275 (2020). DOI: 10.1016/j.jad.2020.06.022

9. Laboratorio de Psicología del Trabajo y Estudios de la Seguridad. Nota de prensa. Madrid: Universidad Complutense de Madrid; 2020. [citado: 30 noviembre 2020]. Disponible en: https://www.ucm.es/estudio-laboratorio-psicologia-trabajo.

10. Gómez-Salgado, J.; Domínguez-Salas, S.; Romero-Martín, M.; Ortega-Moreno, M.; García-Iglesias, J.J.; Ruiz-Frutos, C. Sense of Coherence and Psychological Distress among HealthcareWorkers during the COVID-19 Pandemic in Spain. Sustainability 2020, 12, 6855; doi:10.3390/su12176855

11. Domínguez-Salas, S.; Gómez-Salgado, J.; Andrés-Villas, M.; Díaz-Milanés, D.; Romero-Martín, M.; Ruiz-Frutos, C. Psycho-Emotional Approach to the Psychologi- 
cal Distress Related to the COVID-19 Pandemic in Spain: A Cross-Sectional Observational Study. Healthcare 2020, 8, 190; doi:10.3390/healthcare8030190

12. Ruiz-Frutos, C; Ortega-Moreno, M.; Allande-Cussó, R.; Domínguez-Salas, S.; Dias, A.; Gómez-Salgado, J. Health-related factors of psychological distress during the COVID-19 pandemic among non-health workers in Spain. Safety Science $20121 ; 133,104996$

13. Burdorf, A., Porru, F., Rugulies, R., 2020. The COVID-19 (Coronavirus) pandemic: consequences for 103 occupational health. Scand. J. Work Environ. Health 46 (3), 229-230. https://doi.org/10.5271/sjweh.3893.

14. Moretti A, Menna F, Aulicino M, Paoletta M, Liguori S, Iolascon G. Characterization of Home Working Population during COVID-19 Emergency: A Cross-Sectional Analysis Int. J. Environ. Res. Public Health 2020, 17, 6284; doi:10.3390/ijerph17176284

15. del-Pino-Casado, R.; Espinosa-Medina, A.; López-Martínez, C.; Orgeta, V. Sense of coherence, burden and mental health in caregiving: A systematic review and meta-analysis. J. Affect. Disord. 2019, 242, 14-21.

16. Ruiz-Frutos, C.; Ortega-Moreno, M.; Allande-Cussó, R; Ayuso-Murillo, D.; Domínguez-Salas, S.; Gómez-Salgado, J. Sense of coherence, engagement, and work environment as precursors of psychological distress among non-health workers during the COVID-19 pandemic in Spain. Safety Science 2021, 133, 105033

17. Ruiz-Frutos, C.; Ortega-Moreno, M.; Dias, A.; Bernardes, J.M.; García-Iglesias, J.J.; Gómez-Salgado, J. Information on COVID-19 and Psychological Distress in a Sample of Non-HealthWorkers during the Pandemic Period. Int. J. Environ. Res. Public Health 2020, 17, 6982; doi:10.3390/ijerph17196982

18. Ministerio de Sanidad. Centro de Coordinación de Alertas y Emergencias Sanitarias (CCAES). COVID-19: Situación actual. [citado: 30 noviembre 2020]. Disponible en: https://www.mscbs.gob.es/profesionales/saludPublica/ccayes/alertasActual/nCov/situacionActual.htm

19. World Health Organization. Coronavirus Disease (COVID-19) Pandemic. Situation Report-195. 2020. [citado: 30 noviembre 2020]. Disponible en: https:// www.who.int/docs/default-source/coronaviruse/situation-reports/20200802-covid-19-sitrep-195.pdf?sfvrsn=5e5da0c5_2

20. Elsevier. Novel Coronavirus Information Center. Elsevier's Free Health and Medical Research on the Novel Coronavirus (SARS-CoV-2) and COVID-19. [citado: 30 noviembre 2020]. Disponible en: https://www.elsevier.com/connect/coronavirusinformation-center 